\title{
Análisis comparativo entre una metodología de aprendizaje tradicional y por competencias para alcanzar aprendizajes significativos en calculo diferencial en el primer semestre de agronomía en la ESPOCH
}

Comparative analysis between a traditional learning methodology and competences to achieve significant learning in differential calculus in the first semester of agronomy in the ESPOCH

Marco Hjalmar Velasco Arellano. ${ }^{1}$, Cecilia Teresa Limaico Nieto. ${ }^{2}$ \& John Oswaldo Ortega Castro. $^{3}$

\begin{abstract}
DOI: https://doi.org/10.33262/cienciadigital.v3i2.490

The research work arises as a response to the diverse needs that the first-semester student of the Agronomy Career of the Polytechnic School of Chimborazo in his daily development is experiencing, this makes the research increasingly optimal and efficient to satisfy from certain elementary curiosities to those that arise as a result of a scientific investigation, the same that requires an organized and planned process, contributing to raise the professional level of the teacher to achieve the improvement in the integral education and for the benefit of future generations.

To make the comparative analysis between the traditional methodology and the methodology development of competencies by processes to achieve significant learning of Analytical Geometry has been made a theoretical framework that covers all the reference, concept, importance, methodologies and ideological positioning applied to the present topic, reason for investigation. In addition, it consists of a methodology to be executed, the design of the research, the type of research, the research methods, exposure and discussion of results, the verification of the hypotheses, which allowed obtaining the conclusions.

Finally, being of vital importance the treatment of the Analytical Geometry, it is presented as a proposal some didactic recommendations that are of great practical utility in the treatment of the process of inter-learning, where the mediator propitiates
\end{abstract}

\footnotetext{
${ }^{1}$ Escuela Superior Politécnica de Chimborazo, Facultad de Recursos Naturales. Riobamba, Ecuador. marco.velasco@espoch.edu.ec

${ }^{2}$ Escuela Superior Politécnica de Chimborazo, Facultad de Recursos Naturales. Riobamba, Ecuador. cecilia.limaico@espoch.edu.ec

${ }^{3}$ Escuela Superior Politécnica de Chimborazo, Facultad de Recursos Naturales. Riobamba, Ecuador. john.ortega@espoch.edu.ec
} 
so that the student enjoys what he does, concentrating and participating with interest, secure and confident applications in the Calculus and Topography as sequence materials.

Keywords: Learning - meaningful - methodologies - inter-learning - ideological positioning.

\section{Resumen}

El trabajo investigativo surge como respuesta a las diversas necesidades que el estudiante de primer semestre de la Carrera de Agronomía de la Escuela Superior Politécnica de Chimborazo en su desenvolvimiento cotidiano va experimentando, esto hace que la investigación cada vez sea más óptima y eficaz para satisfacer desde ciertas curiosidades elementales hasta aquellas que surgen como consecuencia de una investigación científica, la misma que requiere de un proceso organizado y planificado, contribuyendo a elevar el nivel profesional del docente para lograr la superación en la educación integral y en beneficio de futuras generaciones.

Para hacer el análisis comparativo entre la metodología tradicional y la metodología desarrollo de competencias por procesos para alcanzar aprendizajes significativos de la Geometría Analítica se ha realizado un marco teórico que abarca todo lo referente, concepto, importancia, metodologías y el posicionamiento ideológico aplicado al presente tema, motivo de investigación. Además, consta de una metodología a ejecutarse, el diseño de la investigación, el tipo de investigación, los métodos de la investigación, exposición y discusión de resultados, la comprobación de las hipótesis planteadas, lo que permitió obtener las conclusiones.

Finalmente, siendo de vital importancia el tratamiento de la Geometría Analítica, se presenta como propuesta algunas recomendaciones didácticas que son de mucha utilidad práctica en el tratamiento del proceso de inter-aprendizaje, donde el mediador propicia para que el estudiante disfrute de lo que hace, concentrándose y participando con interés, seguro y confiado de las aplicaciones en el Cálculo y Topografía como materias de secuencia.

Palabras claves: Aprendizajes - significativos - metodologías - inter-aprendizaje posicionamiento ideológico.

\section{Introducción}

La necesidad imperiosa de mejorar el inter-aprendizaje de la Matemática y en particular de la Geometría Analítica en los estudiantes del primer semestre de la Carrera de Agronomía de la Escuela Superior Politécnica de Chimborazo y el incremento vertiginoso del conocimiento de la ciencia y la tecnología en todas las áreas de la formación profesional, y constituye un reto para que el Docente guie los conocimientos de forma apropiada y emplearla de manera eficiente para la formación integral de la persona la misma que contribuirá al desarrollo del progreso de la sociedad y por ende del país. 
Para lograr un eficiente y eficaz proceso de aprendizaje es indispensable la utilización de los métodos didácticos, estrategias, técnicas, medios y materiales adecuados en concordancia con la complejidad de la materia, impulsando un nuevo enfoque hacia un aprendizaje sin fronteras para asegurar procesos constructivos.

En el presente trabajo de investigación se incluye en su contenido los siguientes capítulos. Capítulo I, consta todo lo referente al marco teórico, es decir abarca todo lo relacionado a las variables que se encuentran inmersas para el desarrollo del proyecto. Capítulo II, la metodología que contiene el diseño, tipos y métodos de investigación. Capítulo III, contiene la exposición y discusión de resultados tanto gráfico como analíticamente. Capítulo IV, contienen las conclusiones y recomendaciones en base a los resultados obtenidos.

El presente trabajo de investigación contribuirá en alguna medida a la formación desarrollando competencias en el proceso educativo. Sin embargo, se debe tender a resolver la problemática educativa, con los propios recursos y potencialidades, recogiendo virtudes, errores u omisiones cometidas para mejorarla y no estancarnos en un nivel. Es necesario crear y recrear en función de nuestras condiciones vigentes, encarándola con fe y esperanza, apoyados por las luces de la ciencia y el eco de la experiencia

- Concepción idealista de Platón: Educar es dar al cuerpo y alma la belleza y toda la perfección de que son susceptibles. (HERRERA, 1999).

- Concepto Dialéctico: Es una ciencia y arte de condiciones en que se refleja la conducta del ser humano con el objeto de construir cada individuo una personalidad desarrollada, integral, social y armónica dentro de las limitaciones naturales, creadora, susceptible de mejoramiento, producción y adoptando el medio, y a la vez capacitar para hacerlo progresar. (USCÁTEGUI, 1965).

La trascendencia del aspecto educativo se manifiesta a través de diferentes puntos de vista así: desde un punto de incorporación, adquisición cognitiva en que se desarrolla y se integra el ser humano en la familia, grupo o clase social de acuerdo a sus capacidades para aprehender constantemente.

Además, creemos relevante ver que la educación viene desde el mismo momento de su concepción del ser humano hasta que cumpla su ciclo de vida en el cual se adapta al medio y sigue patrones de conducta al vincularse con la familia, en grupo y la sociedad, es decir se percibe y concibe la realidad tal como se presenta, se define el proceso de socialización a través de dos subprocesos, socialización e individualización.

Finalmente, la intercomunicación facilita el nexo entre estudiante y docente con el objetivo de orientar, supervisar y evaluar su accionar, al mismo tiempo su interactividad práctica, 
afectiva, emocional e intelectual del individuo que se encuentra en constante relación con los demás. Esto nos ha permitido describir las principales fuentes y aportes de la educación para construir el concepto de competencias.

Con los aportes del desarrollo de competencias integrales deseamos construir a la solución de esta problemática en la guía del proceso de inter-aprendizaje de la Geometría, apropiándonos de los fundamentos de la educación, del constructivismo significativo que posteriormente lo desarrollamos, en tal virtud la educación desde un enfoque por competencias pretende una formación del sujeto para construir - deconstruir - transformar el tejido social que busca un cambio en las estructuras socio comunitarias.

Uno de los grandes aportes de la educación formal para el desarrollo de competencias es buscar superar las metodologías tradicionales basadas en la memorización, acumulación y la repetición mecánica de datos, para privilegiar los procesos cognitivos (percepción, atención, comprensión, inteligencia, y lenguaje) las habilidades cognitivas (interpretación, argumentación y proposición) y la solución de problemas con sentido para para los estudiantes. (TOBÓN, 2006).

La didáctica como disciplina pedagógica (carácter práctico y normativo que tiene por objeto específico la técnica de la enseñanza, esto es la técnica de incentivar y orientar eficazmente a los estudiantes en su aprendizaje), y como didáctica descriptiva en relación a su contenido (es un conjunto sistemático de principios, normas, recursos, procedimientos...específicos que todo docente debe reconocer y saber aplicar para reorientar con seguridad el interaprendizaje de las disciplinas, programas de estudio priorizando competencias y/o objetivos de educación). (BLACIO, 1998).

\section{Comparación entre la Didáctica Tradicional}

\begin{tabular}{|c|c|c|}
\hline \multicolumn{3}{|c|}{ DIDÁCTICA } \\
\hline & DIDÁCTICA TRADICIONAL & DIDÁCTICA POR COMPETENCIAS \\
\hline 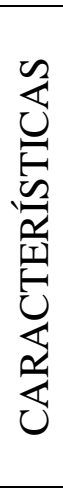 & 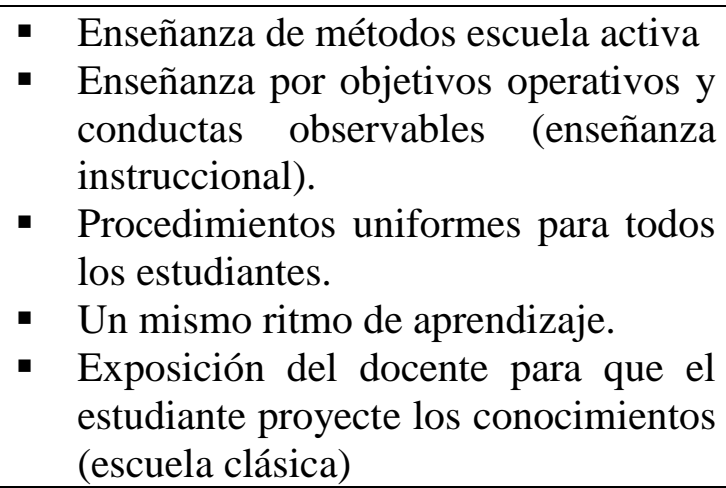 & $\begin{array}{l}\text { - Formación de competencias con base a } \\
\text { problemas comunitarios e intereses } \\
\text { estudiantiles. } \\
\text { - Respeto del ritmo de aprendizaje de las } \\
\text { personas, dadas unas pautas } \\
\text { institucionales y sociales. } \\
\text { - Se emplean estrategias didácticas que } \\
\text { promueven la formación del espíritu } \\
\text { emprendedor, la exploración y la } \\
\text { intervención en el entorno. }\end{array}$ \\
\hline
\end{tabular}




\begin{tabular}{|c|c|c|}
\hline 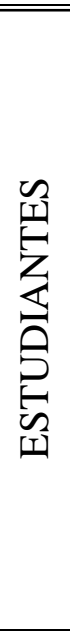 & $\begin{array}{l}\text { ¿A quién se enseña? } \\
\text { Al alumno sumiso, pasivo, receptor y } \\
\text { obediente a los dictámenes de su docente, } \\
\text { su fin es escuchar, repetir sin escuchar } \\
\text { repetir, sin repensar ni construir crítica y } \\
\text { reflexivamente los contenidos aislados y } \\
\text { fragmentados que trasmite su profesor, se } \\
\text { somete con rigidez a todo lo que le dicta e } \\
\text { impone atrofiando su manera de } \\
\text { desarrollarse como persona, se convierte } \\
\text { en receptor de conceptos y contenidos } \\
\text { cuya única meta es aprender lo que le } \\
\text { expone su profesor }\end{array}$ & $\begin{array}{l}\text { ¿A quién se orienta el aprendizaje? } \\
\text { Al estudiante, caracterizado a ser activo, } \\
\text { crítico, reflexivo, investigador y promotor } \\
\text { de la trasformación cultural en la que vive; } \\
\text { se prioriza su desarrollo integral en } \\
\text { coordinación con el docente, es decir } \\
\text { estudiarle desde su multiplicidad de } \\
\text { dimensiones en sus diferentes maneras de } \\
\text { pensar, sentir, actuar y emprender, hacer las } \\
\text { cosas compartiendo, interactuando con la } \\
\text { sociedad. }\end{array}$ \\
\hline & $\begin{array}{l}\text { ¿Quién enseña? } \\
\text { El docente, quien es considerado como } \\
\text { dueño de la materia, es considerado un } \\
\text { déspota y arbitrario, por la imposición y } \\
\text { por la coacción, que no se preocupa por los } \\
\text { problemas y dificultades de los } \\
\text { estudiantes, su meta es lograr la alineación } \\
\text { cultural y la frustración en la formación de } \\
\text { su personalidad, la manera en que se } \\
\text { relaciona es vertical preparando para una } \\
\text { sociedad domesticada y sumisa, en tal } \\
\text { sentido el docente en el aula es como una } \\
\text { máquina que pone en práctica la rutina y } \\
\text { la enseñanza de contextos que no reflejan } \\
\text { su realidad y sus necesidades con la } \\
\text { sociedad, (es partidario del castigo, la } \\
\text { rutina y se cree muy erudito), el docente es } \\
\text { quien planifica, ejecuta y evalúa }\end{array}$ & $\begin{array}{l}\text { ¿Quién orienta al estudiante? } \\
\text { El docente es el que guía, mediador, asesor, } \\
\text { orientador, motivador, líder de la } \\
\text { interacción que fomenta su inter- } \\
\text { aprendizaje priorizando (capacidades } \\
\text { reales, limitaciones, aclarando dudas, } \\
\text { dificultades, programando sus trabajos, } \\
\text { ayudándole a que desarrollen hábitos de } \\
\text { estudio y reflexión). } \\
\text { Busca promover y orientar aprendizajes } \\
\text { significativos de contenidos e impulsar las } \\
\text { macro habilidades competencias de } \\
\text { inteligencia-pensamiento, con el propósito } \\
\text { de que el estudiante sea auto suficiente; el } \\
\text { énfasis no está en los estudiantes ni } \\
\text { tampoco en los docentes sino en la relación } \\
\text { intersistémica de ambos. }\end{array}$ \\
\hline 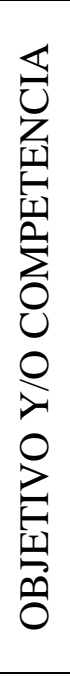 & $\begin{array}{l}\text { ¿Para qué se enseña? } \\
\text { Comprende el objetivo que se persigue al } \\
\text { ser algo meritorio y reconocido como un } \\
\text { ente teórico y remoto que no correlaciona } \\
\text { lo laboral con la vida y el estudio del alma, } \\
\text { su meta es predominar la rutina de } \\
\text { ejercicios repetidos y memorizados, y en } \\
\text { muchos casos, dictados, } \\
\text { descontextualizados de la realidad. } \\
\text { Las competencias son aisladas debido a } \\
\text { que promueven solo el que enseña, es } \\
\text { decir los contenidos, o sea el saber } \\
\text { conocer, notándose evidentemente un } \\
\text { dominio de conductas. }\end{array}$ & $\begin{array}{l}\text { ¿Para qué se orienta al estudiante? } \\
\text { Constituyen objetivos terminales que } \\
\text { actúan como un elemento dinamizador } \\
\text { (dándole sentido valor y dirección a todo el } \\
\text { trabajo de estudiante y profesor... } \\
\text { direccionando las metas propuestas), es } \\
\text { decir respetando sus diferencias } \\
\text { individuales y nivel de maduración en que } \\
\text { se halla el estudiante. Las competencias } \\
\text { integrales promueven la formación integral } \\
\text { correlacionándole el desarrollo de sus } \\
\text { macro habilidades y saberes } \\
\text { aprehenderes. }\end{array}$ \\
\hline
\end{tabular}




\begin{tabular}{|c|c|c|}
\hline 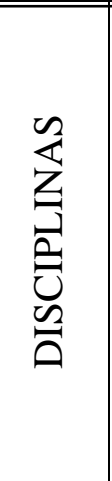 & $\begin{array}{l}\text { ¿Qué se enseña? } \\
\text { Por lo común se enseña asignaturas } \\
\text { aisladas o fragmentadas (que tenían un } \\
\text { valor absoluto y autónomo, es decir } \\
\text { resúmenes extensos, en función de planes } \\
\text { y programas elaborados solo por el } \\
\text { docente, con lo que los estudiantes se } \\
\text { deberían conformar las aprendían al pie de } \\
\text { la letra sin cuestionar, los propios docentes } \\
\text { eran sus esclavos, repitiéndoles fielmente) }\end{array}$ & $\begin{array}{l}\text { ¿Con qué se orienta al estudiante? } \\
\text { Fomentando competencias que promuevan } \\
\text { la polidisciplinaridad, la transversalidad, la } \\
\text { multidimensionalidad en que se entreteje } \\
\text { cada disciplina al formarle integralmente al } \\
\text { estudiante, es decir lograr una formación } \\
\text { auto personal, laboral, empresarial, } \\
\text { económica, cultural, dosificando, } \\
\text { seleccionando en función del estudiante. }\end{array}$ \\
\hline 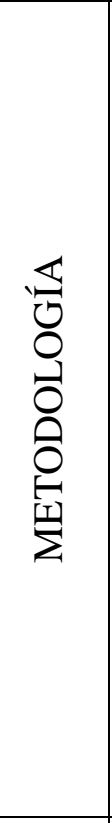 & $\begin{array}{l}\text { ¿Cómo y con qué se enseña? } \\
\text { El cómo enseñar responde a los métodos } \\
\text { pasivos de cada asignatura que en la } \\
\text { práctica son: expositivo, dictado, } \\
\text { conferencia magistral y el interrogatorio, } \\
\text { promovido y elegido por el docente, lo que } \\
\text { produce el desinterés, confusión, apatía al } \\
\text { resolver solo ejercicios de matemática de } \\
\text { una manera mecánica y no problemas, los } \\
\text { métodos cansinos eran la única manera de } \\
\text { exponer su materia de forma abstracta sin } \\
\text { relacionar ni con los intereses ni } \\
\text { necesidades de los estudiantes y la } \\
\text { sociedad. Con respecto al con que enseñar, } \\
\text { su única herramienta era el texto de } \\
\text { estudio y como medio modernizante lo } \\
\text { utilizaban el retroproyector. }\end{array}$ & $\begin{array}{l}\text { ¿Cómo y con qué se orienta al estudiante? } \\
\text { La metodología es activa, participativa y las } \\
\text { técnicas son grupales, teniendo en cuenta } \\
\text { diferencias e intereses de los estudiantes, el } \\
\text { inter-aprendizaje es más centrado en la } \\
\text { metodología activa y por equipo de trabajo } \\
\text { que el estudiante aprenderá, } \\
\text { correlacionándole con las diversas acciones } \\
\text { en que le orientará y organizará el docente } \\
\text { sus saberes y aprehenderes, respetando sus } \\
\text { diferencias individuales, intereses, cultura, } \\
\text { manera de socialización y las estructuras } \\
\text { lógicas del estudiante, de manera que sea } \\
\text { agente de cambio y promotor de las } \\
\text { soluciones de problema reales }\end{array}$ \\
\hline 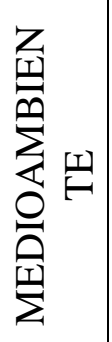 & $\begin{array}{l}\text { ¿Qué se enseña? } \\
\text { No considera las condiciones } \\
\text { fundamentales intra y extra aula en que se } \\
\text { desenvuelve el estudiante como } \\
\text { ventilación, iluminación, separación de la } \\
\text { pizarra a los pupitres. }\end{array}$ & $\begin{array}{l}\text { ¿Dónde se orienta al estudiante? } \\
\text { Prioriza las condiciones en que se } \\
\text { desenvuelve el estudiante y está en contacto } \\
\text { con los problemas de la realidad, del medio } \\
\text { ambiente, en el cual construye su proyecto } \\
\text { ético de vida. }\end{array}$ \\
\hline
\end{tabular}

\section{Pasos metodológicos para la resolución de problemas.}

Es imprescindible desarrollar la capacidad para resolver problemas de la vida práctica, el estudiante debe tener como premisas fundamentales las siguientes: Una sólida base Matemática, desarrollo del pensamiento Matemático, el deseo y la necesidad de hacerlo, tanto en la Matemática como en la Geometría Analítica, existen dos tipos de procedimientos los algorítmicos y los heurísticos, por lo general los pasos lógicos que sobresalen para la resolución de cualquier problema son: 


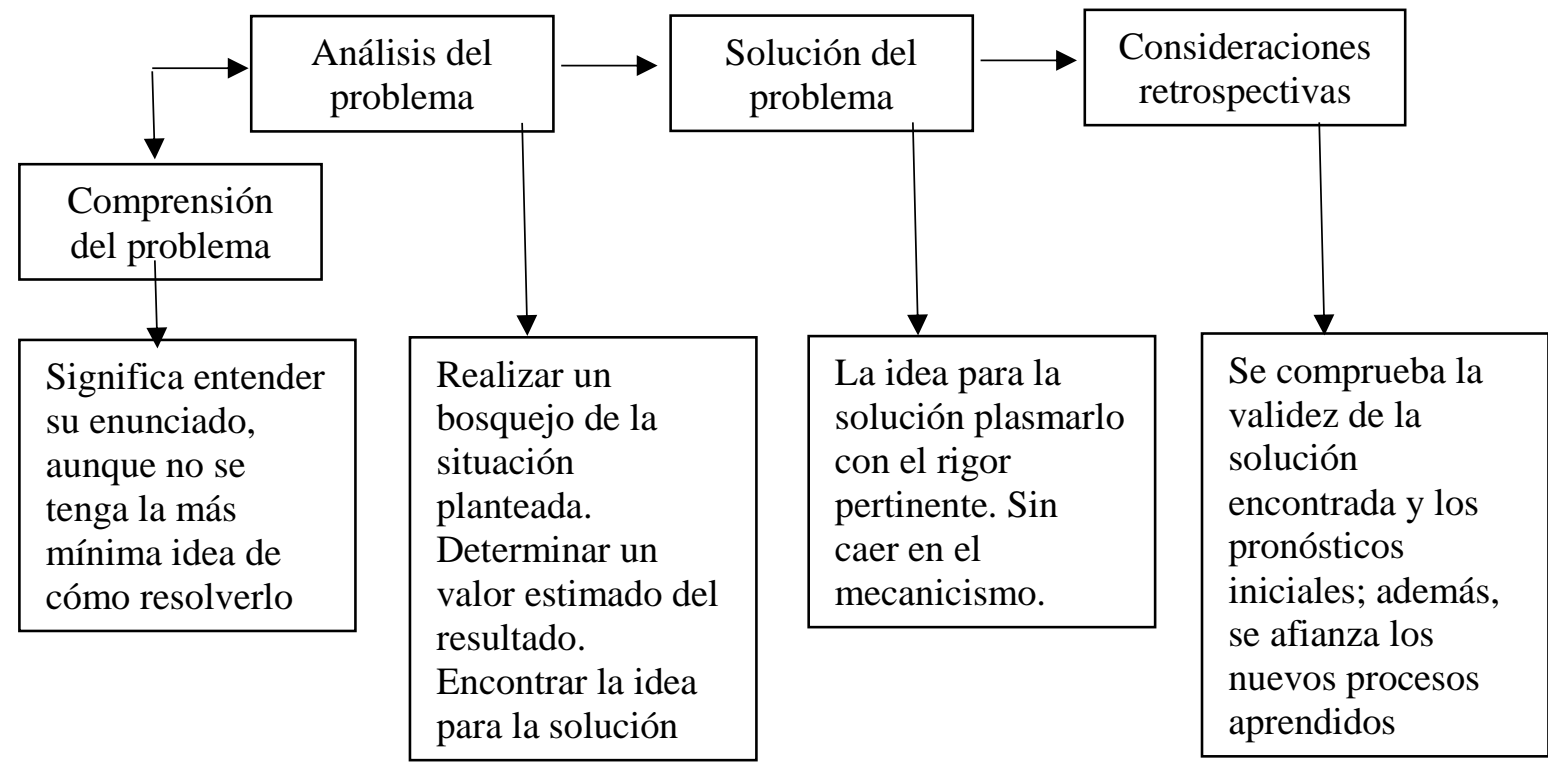

"La Aptitud es predisposición y la competencia es habilidad"

La Aptitud es una predisposición para aprender, pero no es propiamente un aprendizaje, por tratarse de una capacidad fija que caracteriza a los individuos y que se distribuye de manera desigual entre la población determinando las diferencias en el logro educativo. Las aptitudes resultan de la combinación de rasgos mentales, motivacionales y culturales que facilitan a un individuo el aprendizaje en determinada área de desempeño observable. En este sentido la aptitud viene a ser un rasgo innato individual o don natural de cada persona.

La competencia en cambio tiene que ver más con la naturaleza de la mente y la actividad intelectual (proceso cognitivo) comunes a todos los individuos, puesto que existen características cognitivas universales; solo que estas características no dependen exclusivamente de factores intra-psíquicos, ya que las acciones humanas son acciones situadas en un escenario cultural.

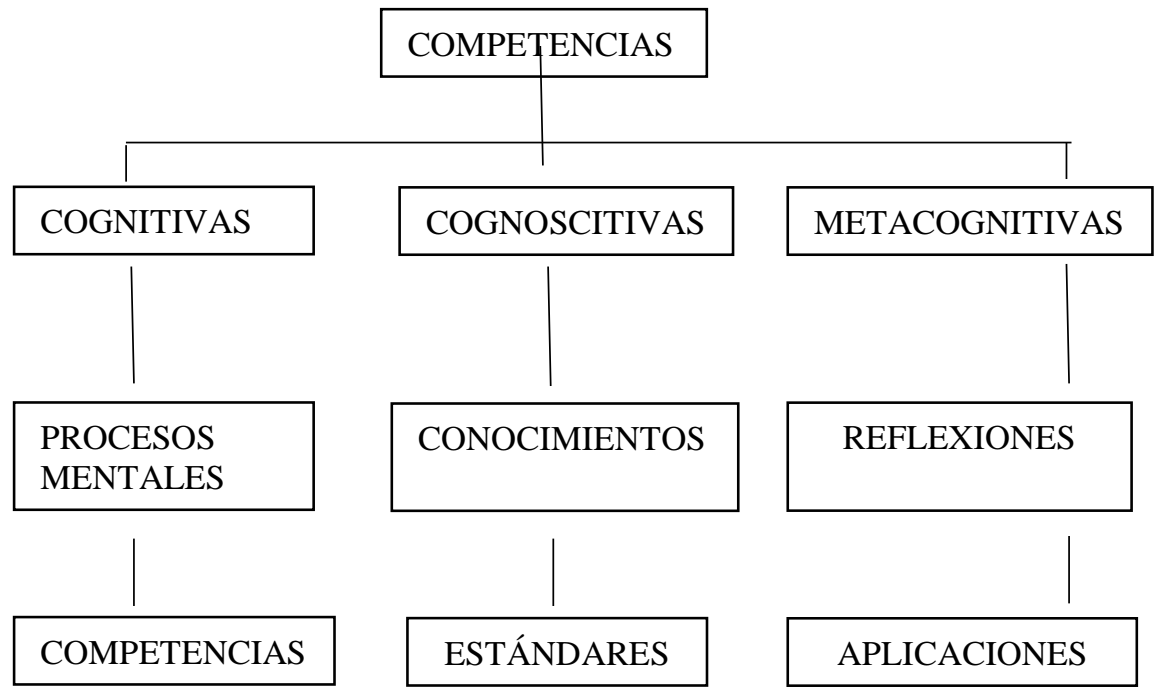


Así hablamos de competencias "cognitivas" para expresar habilidades de pensamiento, de competencias "cognoscitivas" cuando se trata de conceptos y conocimientos específicos de una ciencia o área determinada de un saber o denominado también estándares y una competencia "metacognitiva" alude a los procesos de reflexión sobre el aprendizaje y a la habilidad para aplicar conceptos y temas a la vida profesional y cotidiana. (FLORES, 1989)

\section{Temas propuestos de la Geometría Analítica.}

1. Sistemas de Coordenadas

1.1. Segmento rectilíneo dirigido.

1.2. Sistema coordenado lineal.

1.3. Distancia entre dos puntos.

1.4. Ecuaciones de la recta.

1.5. Cálculo de la Pendiente.

1.6. Cálculo del ángulo de inclinación.

1.7. Área de un triángulo

1.8. Demostración gráfica y aplicación en software

2. Gráfica de una ecuación y lugares geométricos.

2.1. Intersección con los ejes coordenados.

2.2. Criterios de simetría.

2.3. Extensión de una curva.

2.4. Asíntotas.

2.5. Tabla de valores, gráfica y aplicación de software.

3. Ecuación de la Circunferencia.

3.1. Forma general de la ecuación de una circunferencia.

3.2. Familia de circunferencias que pasan por la intersección de dos circunferencias.

3.3. Tangente a una circunferencia.

3.4. Gráfica y aplicación en software.

4. La parábola.

4.1. Ecuación de la parábola de vértice en el origen.

4.2. Ecuación de la parábola de vértice $(\mathrm{h}, \mathrm{k})$ y eje paralelo a un eje coordenado.

4.3. Ecuación general de una parábola.

4.4. Gráfica y aplicación en software.

5. La Hipérbola

5.1. Ecuación ordinaria.

5.2. Asíntotas de una hipérbola.

5.3. Hipérbola equilátera o rectangular.

5.4. Ecuación en forma general.

5.5. Ecuación de la tangente a una hipérbola.

5.6. Gráfica y aplicación en software.

(LEHMAN, 1972) - (LEITHOLD, 1992) 
Es cierto que la Matemática y en especial la Geometría Analítica se aprende haciendo ejercicios, debido a que proporciona destrezas que son indispensables para el desarrollo adecuado del pensamiento, en los ejercicios se concreta la aplicación de conceptos, teoremas y nuevos conocimientos.

Todo ejercicio matemático constituye una exigencia, la cual presenta tres componentes:

a. El objetivo: Transforma una situación inicial (elementos dados), en una situación final (elementos buscados).

b. El contenido: Elementos de la materia (conceptos, teoremas, procedimientos, algoritmos) y su correspondencia con situaciones extra-matemáticas.

c. Las condiciones para las acciones: Conocimientos que posee el alumno y el grado de dificultad de las acciones, las cuales pueden ser, identificar, comparar, calcular, demostrar, clasificar, reconocer, describir, aplicar, fundamentar, etc.

En los estudiantes de Primer Semestre de la Carrera de Agronomía de la Facultad de Recursos Naturales de la Escuela Superior Politécnica de Chimborazo, se incentiva la gran importancia de cada uno de los elementos, procesos algebraicos y representaciones gráficas de manera manual y por medio de la utilización de software de la Geometría Analítica para las aplicaciones en el Cálculo y más que todo en la Topografía, conocimientos que permitirán la seguridad en los estudiantes para comprender bien la ejercitación y de tal manera confiar que los resultados obtenidos sean de manera óptima plasmados en las planimetrías o en los software de diseño planimétrico. Esto permite que cada nuevo contenido se sistematice y puedan combinarse con los elementos precedidos, así se retroalimenta constantemente, ya que los contenidos aislados entorpecen las nuevas estructuras mentales.

\section{Metodología}

\section{Diseño de la investigación}

Con el objetivo de indagar el problema investigativo formulado y plantear la prueba de la hipótesis, hemos elegido el diseño cuasi-experimental, caracterizado por ser específico; su utilidad nos facilitará someter a experimentación y buscar interrelación al manipular deliberadamente la variable independiente; la metodología para el desarrollo de competencias por procesos con el grupo experimental (estudiantes de Primer Semestre paralelo 3) y la metodología tradicional con el grupo de control (estudiantes de Primer Semestre paralelo 1); esto nos conlleva a determinar su efecto y relación con la variable dependiente el nivel de aprendizaje significativo en Geometría Analítica.

\section{Tipo de investigación}

Puesto que la investigación se realiza durante un determinado intervalo de tiempo, el diseño es también de tipo longitudinal de análisis evolutivo. 
Debido a que con el trabajo de investigación buscamos averiguar la influencia de una variable (aplicaciones móviles, software) sobre otra (rendimiento académico), esta es de tipo correlacional, porque busca conocer el nivel de relación entre las variables.

La Población está constituida por los estudiantes de primer semestre 1 y 2 de la Facultad de Recursos Naturales de la Carrera de Agronomía de la Escuela Superior Politécnica de Chimborazo, en el periodo académico abril - agosto 2016.

\section{Método de investigación}

En concordancia con el problema de investigación y las características de la disciplina de Geometría Analítica utilizaremos métodos lógicos que toman en cuenta el razonamiento del estudiante - docente y actividades que nos contribuirán a desarrollar competencias en el proceso de aprendizaje significativo, son el método inductivo - deductivo que son parte del método.

La aplicación del método científico nos da la respuesta, como investigaremos, sus consideraciones lógicas, se refiere a la estructura lógico formal de las ciencias exactas "Matemática" y "Lógica Dialéctica", estar en contacto el objeto sujeto de investigación. Sus etapas principales que ejecutaremos son:

- Observación y determinación del problema consta de: identificación, delimitación contextualización y formulación presentadas en el proyecto.

- Planteamiento de hipótesis se debe revisar la bibliografía y construcción de un modelo teórico que refleja la respuesta teórica que hemos dado a los objetivos investigados previamente. Todos aspectos permitieron formular la hipótesis y elaborar el esquema de relaciones del cuasi-experimento.

- Comprobación y/o prueba de hipótesis, ha constituido: diseño, ejecución de la prueba, sistematización y análisis de datos e inferencia de las conclusiones.

\section{Técnicas e instrumentos de recolección de datos}

\begin{tabular}{ll}
\hline \multicolumn{1}{c}{ Técnica } & \multicolumn{1}{c}{ Instrumento } \\
\hline Test & Prueba objetiva \\
La Observación & Guía o ficha de observación \\
\hline
\end{tabular}

\section{Población y muestra}

Puesto que el número de estudiantes en cada uno de los paralelos es menor que 40, no se toma una muestra y la investigación se realiza con todos los integrantes independientemente. 


\section{Técnicas de procedimientos para el análisis de resultados}

Una vez recopilados los datos a través de los instrumentos determinados se procederá a la tabulación, análisis y prueba de hipótesis. Los resultados se mostraran mediante tablas, cuadros, gráficos y la prueba de hipótesis por medio del t-student.

\section{Análisis e interpretación de los resultados generales}

De los resultados particulares relativos a cada tema de estudio sobre el nivel de aprendizaje de la Geometría Analítica en los estudiantes de Primero semestre paralelos 1 y 3 semestre, utilizando la metodología desarrollo de competencias por procesos que pertenece al grupo experimental, es superior al nivel de aprendizaje utilizando la metodología tradicional del grupo de control, observando claramente en los gráficos estadísticos, que el grupo experimental tiene mejor promedio que el grupo de control, aunque no en la misma proporción, siendo más marcada la diferencia en cuanto al tema sobre el desarrollo de competencias de la Geometría Analítica y sus aplicaciones en el Cálculo y Topografía.

Como el estudio se ha hecho sobre toda la población, podemos asegurar que la aplicación del desarrollo de competencias por procesos del aprendizaje de la Geometría Analítica, tiene una incidencia positiva en el rendimiento académico en comparación al grupo de control que no usaron dichos procesos de aprendizaje por competencias.

Pero es necesario medir esta diferencia respecto al promedio total de ambos grupos, esto lo indicamos en las tablas, gráficos, los cuales servirán también en la aceptación o rechazo de la Hipótesis, es decir:

\subsection{Datos del grupo de control (Paralelo “3”)}

\begin{tabular}{ccccc}
\hline$y_{i}$ & $x_{i}$ & $\boldsymbol{\%}$ & $\begin{array}{c}\boldsymbol{\%} \\
\text { Acumulado }\end{array}$ & $\boldsymbol{n}_{x_{i}}$ \\
\hline 4,00 & 0 & 0 & 0 & 23 \\
5,00 & 3 & 13 & 13 & 23 \\
6,00 & 6 & 26 & 39 & 20 \\
7,00 & 5 & 22 & 61 & 14 \\
8,00 & 4 & 17 & 78 & 9 \\
9,00 & 5 & 22 & 100 & 5 \\
10,00 & 0 & 0 & 100 & 0 \\
Total & $\sum x_{i}=23$ & 100,00 & & \\
\hline
\end{tabular}




\begin{tabular}{lc}
\hline \multicolumn{1}{c}{ Valores estadísticos } & Total \\
\hline \# Estudiantes & 23,00 \\
Media & 7,09 \\
Mediana & 6,00 \\
Moda & 6,00 \\
Desv. Estándar & 0,53 \\
Varianza & 0,28 \\
Rango & 6,00 \\
Mínimo & 4,00 \\
Máximo & 10,00 \\
\hline
\end{tabular}

\subsection{Datos del grupo experimental (Paralelo "1")}

\begin{tabular}{|c|c|c|c|}
\hline$y_{i}$ & $x_{i}$ & $\%$ & $\begin{array}{c}\% \\
\text { Acumulado }\end{array}$ \\
\hline 4,00 & 0 & 0 & 0 \\
\hline 5,00 & 2 & 8 & 8 \\
\hline 6,00 & 4 & 17 & 25 \\
\hline 7,00 & 6 & 25 & 50 \\
\hline 8,00 & 7 & 29 & 79 \\
\hline 9,00 & 4 & 17 & 96 \\
\hline 10,00 & 1 & 4 & 100 \\
\hline \multirow[t]{11}{*}{ Total } & $\sum x_{i}=24$ & 100,00 & \\
\hline & \multicolumn{2}{|c|}{ Valores estadísticos } & Total \\
\hline & \multicolumn{2}{|c|}{ \# Estudiantes } & 24,00 \\
\hline & \multicolumn{2}{|c|}{ Media } & 7,42 \\
\hline & \multicolumn{2}{|c|}{ Mediana } & 7,00 \\
\hline & \multicolumn{2}{|c|}{ Moda } & 8,00 \\
\hline & \multicolumn{2}{|c|}{ Desv. Estándar } & 0,53 \\
\hline & \multicolumn{2}{|c|}{ Varianza } & 0,29 \\
\hline & \multicolumn{2}{|c|}{ Rango } & 6,00 \\
\hline & \multicolumn{2}{|c|}{ Mínimo } & 4,00 \\
\hline & \multicolumn{2}{|c|}{ Máximo } & 10,00 \\
\hline
\end{tabular}

\section{Hipótesis}

Ho: $\overline{X_{1}}=\overline{X_{2}}$

La Hipótesis Nula expresa: El nivel de aprendizaje de la geometría Analítica en los estudiantes de los Primeros Semestres paralelos 1 y 3 de la Carrera de Agronomía de la Escuela Superior Politécnica de Chimborazo, utilizando la metodología desarrollo de competencias por procesos no es superior al nivel de aprendizaje utilizando la metodología tradicional. 
$\mathbf{H}_{\mathrm{i}}: \overline{X_{1}} \neq \overline{X_{2}}$

El nivel de aprendizaje de la geometría Analítica en los estudiantes de los Primeros Semestres paralelos 1 y 3 de la Carrera de Agronomía de la Facultad de Recursos Naturales de la Escuela Superior Politécnica de Chimborazo, utilizando la metodología desarrollo de competencias por procesos si es superior al nivel de aprendizaje utilizando la metodología tradicional.

\subsection{Aplicación del T-Student para la aceptación o nulidad de la hipótesis}

\begin{tabular}{lr}
\hline Valoración & Cantidad \\
\hline Nivel de significancia $\alpha$ (a una cola) & 0,05 \\
Nivel de significancia $\frac{\alpha}{2}$ (a dos colas) & 0,025 \\
Media $\overline{X_{1}}$ grupo de control & 7,09 \\
Media $\overline{X_{2}}$ grupo experimental & 7,42 \\
Desviación Estándar $s_{1}$ & 0,53 \\
Desviación Estándar $s_{2}$ & 0,53 \\
Varianza $s_{1}^{2}$ & 0,28 \\
Varianza $s_{2}^{2}$ & 0,29 \\
$n_{1}$ Número de estudiantes paralelo 1 & 23 \\
$n_{2}$ Número de estudiantes paralelo 2 & 24 \\
\hline
\end{tabular}

- Grados de libertad $(v)$

$$
\begin{aligned}
& v=n_{1}+n_{2}-2 \\
& v=23+24-2 \\
& v=45
\end{aligned}
$$

- T-Student (calculado)

$$
\begin{aligned}
& t_{c}=\frac{\overline{X_{1}}-\overline{X_{2}}}{\sqrt{\frac{\left(n_{1}-1\right) s_{1}^{2}+\left(n_{2}-1\right) s_{2}^{2}}{n_{1}+n_{2}-2}\left(\frac{1}{n_{1}}+\frac{1}{n_{2}}\right)}} \\
& t_{c}=\frac{7,09-7,42}{\sqrt{\frac{(22)(0,53)^{2}+(23)(0,53)^{2}}{45}\left(\frac{1}{23}+\frac{1}{24}\right)}} \\
& t_{c}=-2,13
\end{aligned}
$$


- T-student (Tabla de la prueba del " $t$ ”)

$$
\begin{aligned}
& t_{t}=t_{0,025 \rightarrow 45}=2,02 \\
& -2,02 \leq t_{c} \leq 2,02 \quad \text { (a dos colas) } \\
& -2,02 \leq-2,13 \leq 2,02
\end{aligned}
$$

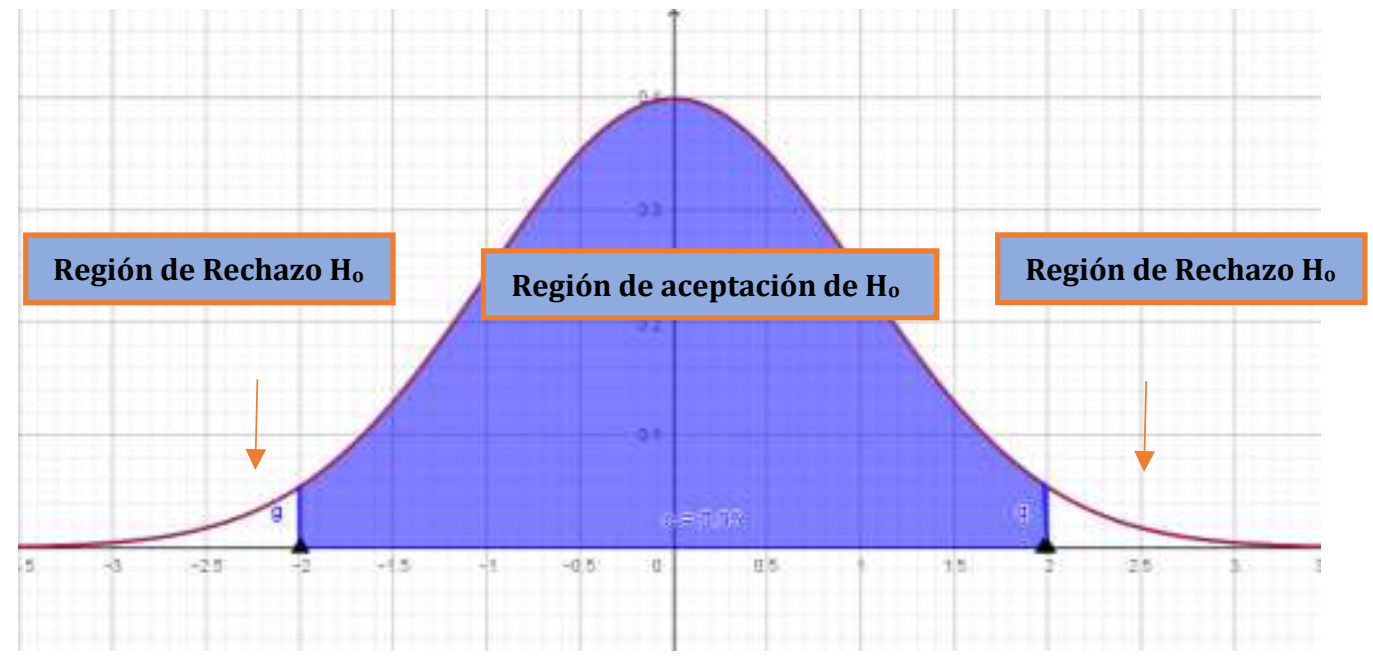

Fuente: Actas de calificaciones de la Catedra de Cálculo (CA-FRN)

Elaborado por: MsC. Marco Velasco

\section{Resultados}

El promedio del nivel de aprendizaje significativo obtenido en la Catedra de Geometría Analítica mediante el empleo de la metodología desarrollo de competencias, es totalmente significativo al nivel de aprendizaje utilizando la metodología tradicional del primer semestre paralelo "3" (grupo de control) con respecto al primer semestre paralelo "1" (grupo experimental) de la Carrera de Agronomía de la Facultad de Recursos Naturales de la Escuela Superior Politécnica de Chimborazo.

Además el análisis estadístico que se realizó en la distribución Gaussiana es a dos colas de tal manera que el resultado para la aceptación de la Hipótesis alterna es $t_{c}<t_{t}$ es decir $-2,13<2,02$, entonces se rechazó la Hipótesis Nula $H_{o}$ y aceptamos la Hipótesis Alternativa $H_{i}$ es decir que el nivel de aprendizaje de los estudiantes empleando la metodología desarrollo de competencias por procesos es superior al nivel de aprendizaje de los estudiantes que se utilizó la metodología tradicional. 


\section{Conclusiones}

- La metodología por competencias desarrolla habilidades y destrezas para el aprendizaje de la Geometría Analítica más eficazmente que la metodología tradicional.

- La definición y la conceptualización se complementan desarrollando en el estudiante la capacidad de resolver el problema en forma analítica y gráfica. En la metodología tradicional solamente se utiliza el procedimiento matemático.

- La metodología por procesos permite elaborar material concreto que el docente maneja o manipula para aprender a hacer y saber.

- La metodología o modelo de aprendizaje por competencias, permite al docente exponer con claridad lo aprehendido, desarrollando su apropiación crítica.

\section{Referencias Bibliografícas}

BARNETT. R. Precálculo Funciones y Gráficas. McGraw-Hill. $4^{\text {ta }}$ edición. México.1982.

BLACIO. G. Didáctica General. ESPE. Quito - Ecuador 1998.

HERRERA. E. Filosofía e história de la Educación. UCE. Quito - Ecuador 1999.

LEHMAN.Ch. Geometría Analítica. UTEHA. México.1972.

LEITHOLD. L. Cálculo con Geometría Analítica. Oxford 7ª edición. México. 1992.

OCHOA. R. Investigación Pedagógica y Educativa. Colombia.1989.

SÁNCHEZ. P. El Proceso de enseñanza aprendizaje. ICE de la UCM. Madrid - España 2004.

TOBÓN. S. Aspectos Básicos de la Formación Basada en Competencias. Talca. Proyecto. Mesesup 2006.

USCÁTEGUI. E. Pedagogía Científica Editorial Universitaria. Quito - Ecuador 1965.

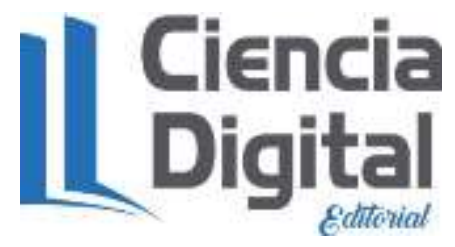




\section{PARA CITAR EL ARTÍCULO INDEXADO.}

Velasco M., Limaico C. \& Ortega J. Factores de riesgo de fracturas de radio en niños menores de 12 años: Resultados de estudio piloto, Revista electrónica Ciencia Digital 3(2), 735-750. Recuperado desde:

http://cienciadigital.org/revistacienciadigital2/index.php/CienciaDigital/article/view/263/56 7

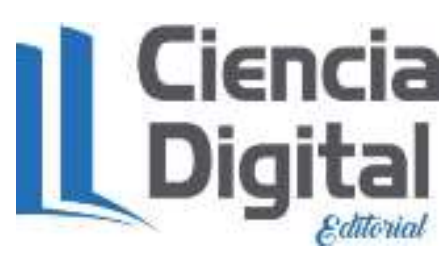

El artículo que se publica es de exclusiva responsabilidad de los autores y no necesariamente reflejan el pensamiento de la Revista Ciencia Digital.

El artículo queda en propiedad de la revista y, por tanto, su publicación parcial y/o total en otro medio tiene que ser autorizado por el director de la Revista Ciencia Digital.
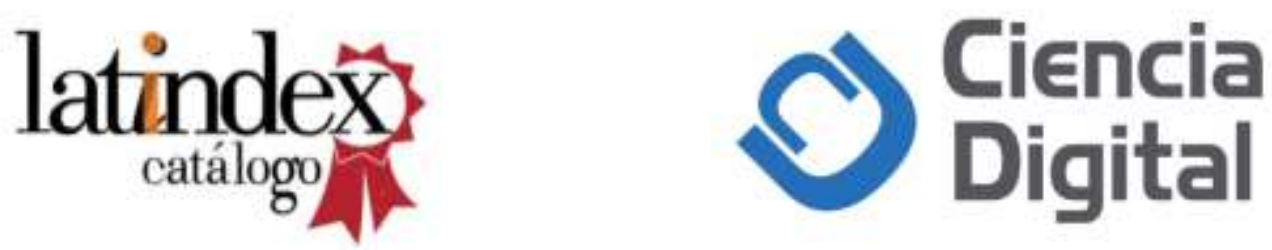